\title{
The Effects of Footwear on Squat Movements
}

\author{
Joshua W. Cohen ${ }^{1}$, Philip Lee ${ }^{1}$, Jessa Buchman-Pearle ${ }^{1}$ \\ ${ }^{1}$ University of Western Ontario
}

\begin{abstract}
The purpose of this study was to compare the effects of different footwear (bare feet, flat-soled shoes, and weightlifting shoes) on balance during a barbell back squat in novice and advanced participants. It was hypothesized that i) weightlifting shoes would increase balance performance in the anterior-posterior (AP) and medial-lateral (ML) directions, and ii) advanced participants would demonstrate increased balance performance in the AP and ML direction compared to novice participants. 17 participants $(9$ novice and 8 advanced) performed 3 trials of a single squat repetition, using a 45lb Olympic standard barbell on a force plate, with bare feet, flat shoes, and weightlifting shoes. Squat 1 and 2 were used as warm-up repetitions and data was collected during squat 3 of each condition. Participants were characterized as either novice or advanced based on the number of training sessions they performed squats each week. Novice participants squatted with a barbell less than once per week, and advanced squatted with a barbell more than once per week. Balance performance was quantified by the percentage of time the centre of pressure (CoP) had the smallest displacement from the average CoP location. A chi-square test was performed to determine the statistically significant changes in CoP displacement between the 3 footwear conditions; accordingly, the post hoc significance interval was calculated to P0.0167). Using a 2-way ANOVA, no significant differences were found between novice and advanced participants' root mean square values for normalized $\mathrm{CoP}$ in the AP or ML directions $(\mathrm{P}>0.05)$. Participants during the weightlifting shoe condition were found to have better regulation of balance compared to bare feet or flat shoes conditions. Additionally, it was found that novice and advanced participants did not regulate balance differently from each other during the squat movement.
\end{abstract}

KEYWORDS: biomechanics, balance regulation, strength and conditioning

\section{Introduction}

The squat is one of the most prevalent compound exercises used in athletic strength training, as well as rehabilitation $(1,2)$. Performing the squat requires muscle activation throughout the lower extremity, specifically, the gluteals, quadriceps, hamstrings, gastrocnemius, and tibialis anterior (3). Due to the sequential and simultaneous contractions of these muscles, balance is a fundamental aspect of the squat movement (4). Balance is defined as the regulation of an individual's body to maintain stability (5). To accomplish this, the centre of pressure (CoP) drives the centre of mass (CoM) within the boundaries of the base of support (BoS) (6). CoP location can be calculated from the ground reaction forces produced by an individual standing on a force plate (5).

Fundamentally, there are three postural changes to regulate balance: increasing the area of the BoS, utilizing ankle and hip musculature. Anterior-posterior (AP) balance regulation is accomplished by plantar flexors (ankle strategy), as well as hip flexors and extensors (hip strategy). The ankle strategy is used more often, unless a large perturbation is encountered. Medial-lateral (ML) regulation is accomplished at the hips by abductors and adductors $(7,8)$. In addition to the postural changes, balance is maintained by the integration of three afferent systems in the central nervous system
(CNS): visual, vestibular, and proprioceptive (9). It has been found that regulation of balance is better barefoot as this provides more feedback to the cutaneous system (10).

The increase in balance regulation with the utilization of the cutaneous system may also be true when performing squats in bare feet (BF). Changes in kinematics occur as different musculature is recruited throughout the squat; different footwear causes these musculature changes (11). In weightlifting shoes (WLS) (Figure 1A) a rigid midsole and heel lift increases the amount of ankle plantar flexion (12). By increasing ankle plantar flexion, muscular recruitment will change during the squat movement, resulting in increased plantar flexor activation (13). As the ankle strategy relies on plantar flexors, it is postulated that the increase in plantar flexor activation will help regulate balance.

Previous research has demonstrated that cushioned footwear (Figure 1B) negatively affects balance performance (14). Body weight applies a downward force on the shoe resulting in compression of the cushioning. While squatting, this creates a less stable base, thereby compromising performance and safety throughout the exercise (15). Flat-soled shoes (FS) (Figure 1C) may alleviate the negative effects of cushioning.

Previous research has demonstrated that different footwear can result in kinematic changes 
during the squat movement $(12,14,16)$. However, few studies have examined the effects of footwear on the kinetics driving these kinematic changes during the squat movement. The purpose of this study was to compare the kinetics of the barbell back squat in novice and advanced participants wearing three types of footwear conditions; BF, FS, and WLS. It was hypothesized that i) WLS would increase balance performance in the $A P$ and $M L$ directions, and ii) advanced participants would demonstrate increased balance performance in the AP and ML direction compared to novice participants.

A) Weightlifting Shoes

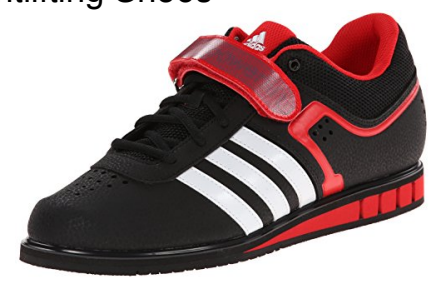

B) Athletic Footwear

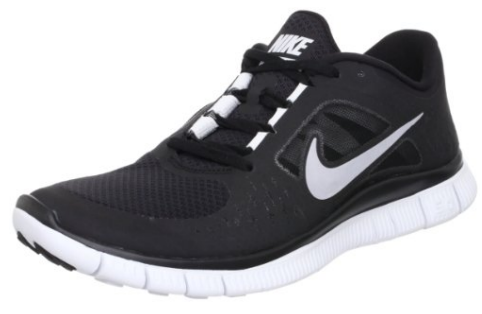

C) Flat-soled Shoes

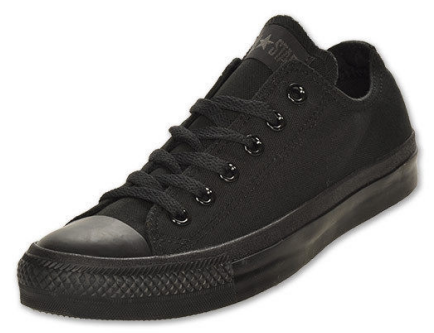

Figure 1. Pictures of different footwear.

\section{Methods \\ Participants}

This study was approved by the University of Western Ontario's Health Sciences Research Ethics Board, and all participants provided written informed consent. They were recruited by posters distributed throughout the University of Western Ontario. Participants were included if they were between 1824 years of age, male, English speaking, and able- bodied. The exclusion criteria were if they had sustained any lower extremity injuries 3 months prior to testing, or any other musculoskeletal disorders.

17 male participants (mean age 20.8 years, $\mathrm{SD}=1.3$ years; mean height $180.9 \mathrm{~cm}, \mathrm{SD}=5.9 \mathrm{~cm}$; mean weight $83.4 \mathrm{~kg}, \mathrm{SD}=11.1 \mathrm{~kg}$ ) volunteered in the study. All participants had previously squatted with an 45lb Olympic standard barbell. Participants were classified as either novice or advanced, based on the number of training sessions in which they performed squats each week. Novice participants squatted with a barbell less than once per week, and advanced squatted with a barbell more than once per week.

\section{Protocol}

Participants wore three footwear conditions: i) BF, ii) FS, and iii) WLS. 3 squats were performed for each condition; squat 1 and 2 were used as warmup, and data was collected during squat 3 . Pilot testing determined that the third squat provided the smallest CoP displacement, indicating their best trial. Condition orders were counterbalanced, and shoes in the participant's size were provided.

Participants stood on the force plate in their preferred squat stance and a 45lb Olympic standard barbell was placed on their back. Tape was used to outline each participant's BoS and participants stood at this location for each of their trials. Participants were allotted 5 seconds to complete the full squat movement, descending to 90 degrees of knee flexion and ascending to stand. 5 seconds elapsed between the trials.

\section{Equipment}

A Kistler force plate (Kistler 9287 B, Kistler Holding AG, Winterthur, Switzerland) was used to measure the ground reaction forces and moments of the Cartesian coordinate system. Force plate voltage signals were amplified with an analogue-to-digital conversion board, and recorded with a custom LabVIEW software. Voltages were converted to forces and moments using the LabVIEW software. These data were processed in Microsoft Excel 2016 and PRISM 7. The Adidas Powerlifts, in the participant's size, were provided for the WLS condition, and Converse Chuck Taylors, in the participant's size, were provided for the FL condition. A 45lb Olympic standard barbell was used for each trial.

\section{Data analysis}

Each participant's squat varied in time as making participants perform a standardized squat could have resulted in unnatural movement. 
Therefore, the data file was trimmed, outlined in the calculations section below, to standardize each squat movement. CoM velocity was calculated using the impulse-momentum relationship (Calculation 1 and Calculation 2). CoM velocity and time were multiplied to determine CoM displacement (Calculation 3). The slope of the CoM displacement was then calculated (Calculation 4). When CoM displacement slope is positive, the participant was moving up; when the slope is negative, the participant was moving down. When the slope was equal to 0 , the participant had completed the movement, and the data file was cut. These calculations were verified with visual analysis of the graphs (Figure 2).

No participants were excluded from the analysis. A chi-square test was performed to determine the statistically significant changes in CoP displacement between the 3 footwear conditions. Accordingly, a post hoc was completed, in which the significance interval was calculated and set to $\mathrm{P}<0.0167$ (Calculation 6).

2-way ANOVA was performed to compare novice and advanced RMS values in the AP and ML directions for the normalized CoP data. Significance was set to $\mathrm{P}<0.05$.

\section{Calculations}

1. Due to each participant's squat varying in time, data points for when individuals were completing the squat movement were extracted by determining when CoM was moving upwards (positive slope), downwards (negative slope), or at starting position (0):

$$
J=F * t
$$

where $J$ is impulse, $F$ is force, and $t$ is time.

$$
v_{C o M}=\frac{F * t}{m},
$$

where $v_{C o M}$ is CoM velocity, and $m$ is mass.

$$
d_{1}=v_{\text {avg }} * 0.01+d_{0},
$$

where $d_{1}$ is CoM displacement from starting position, $v_{\text {avg }}$ is average velocity in 0.01 seconds, and $d_{0}$ is the previous CoM displacement.

$$
s=\frac{\Delta d}{0.01}
$$

where $s$ is the slope of CoM displacement $\Delta d$ is the difference between subsequent displacements.

2. CoP in AP and ML direction was calculated by:

$$
\begin{aligned}
\operatorname{CoP}_{A P} & =-\frac{M_{y}+c F_{x}}{F_{z}}, \\
\operatorname{CoP}_{M L} & =\frac{M_{x}-c F_{y}}{F_{z}},
\end{aligned}
$$

where $M$ is moment, $c$ is origin location of the force plate coordinate system, and $F$ is ground reaction force.

3. Data was normalized via the following:

$$
\operatorname{CoP}_{\text {normal }}=\operatorname{CoP}_{\text {original }}-\operatorname{CoP}_{\text {mean }} .
$$

4. Percentage of time normalized CoP spent in each radius within the BoS was calculated by:

$$
\% t=\frac{n_{\text {radius }}}{n_{\text {total }}} \times 100 \% \text {, }
$$

where $\% t$ is percentage of time spent by normalized $\mathrm{CoP}, n_{\text {radius }}$ is number of data points within a specific radius $\left(10 \mathrm{~mm}, 20 \mathrm{~mm}\right.$, and $n_{\text {total }}$ is total number of data points.

5. Root Mean Square (RMS) in AP and ML directions was calculated by:

$$
R M S_{A P}=\sqrt{\frac{\sum_{i=0}^{n} x_{i}^{2}}{n}},
$$

$$
R M S_{M L}=\sqrt{\frac{\sum_{i=0}^{n} y_{i}^{2}}{n}},
$$

where $n$ is number of subjects ( $n_{\text {novice }}=9, n_{\text {advanced }}=$ 8), $x$ or $y$ is each subjects normalized CoP displacement.

6. Post hoc significance level was determined by calculating Bonferroni's correction factor:

$$
\beta=\frac{0.05}{n}
$$

where $\beta$ is Bonferroni's correction, and $n$ is number of conditions compared $(n=3)$. 


\section{Results}

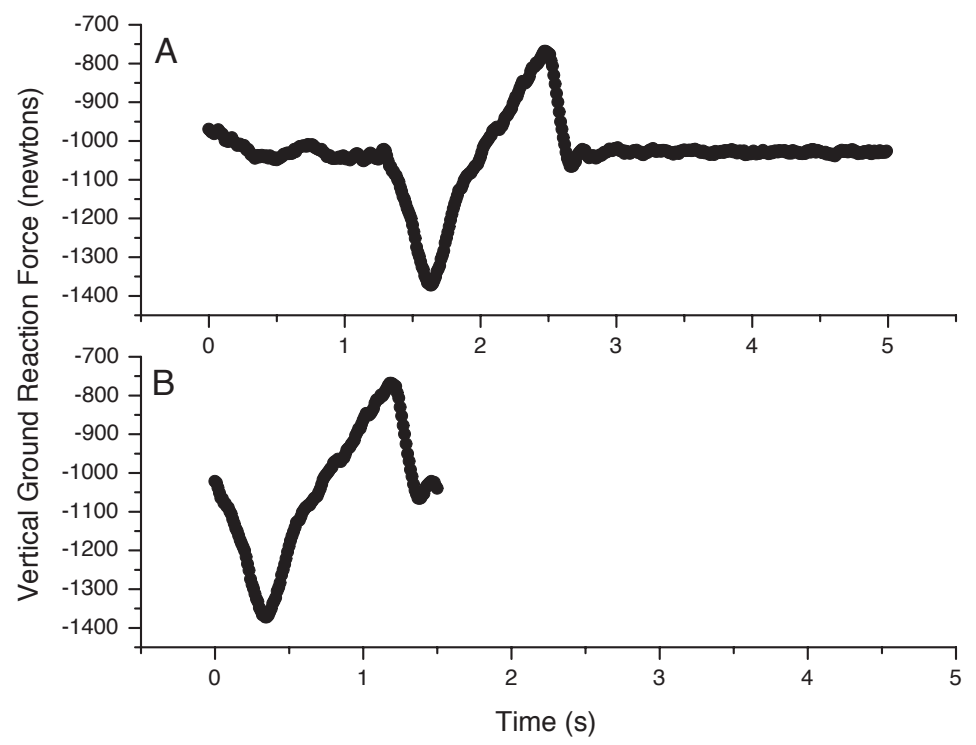

Figure 2: Data analysis of vertical ground reaction force of a single squat trial. A denotes the raw data file before our analysis, B denotes the trimmed data file.
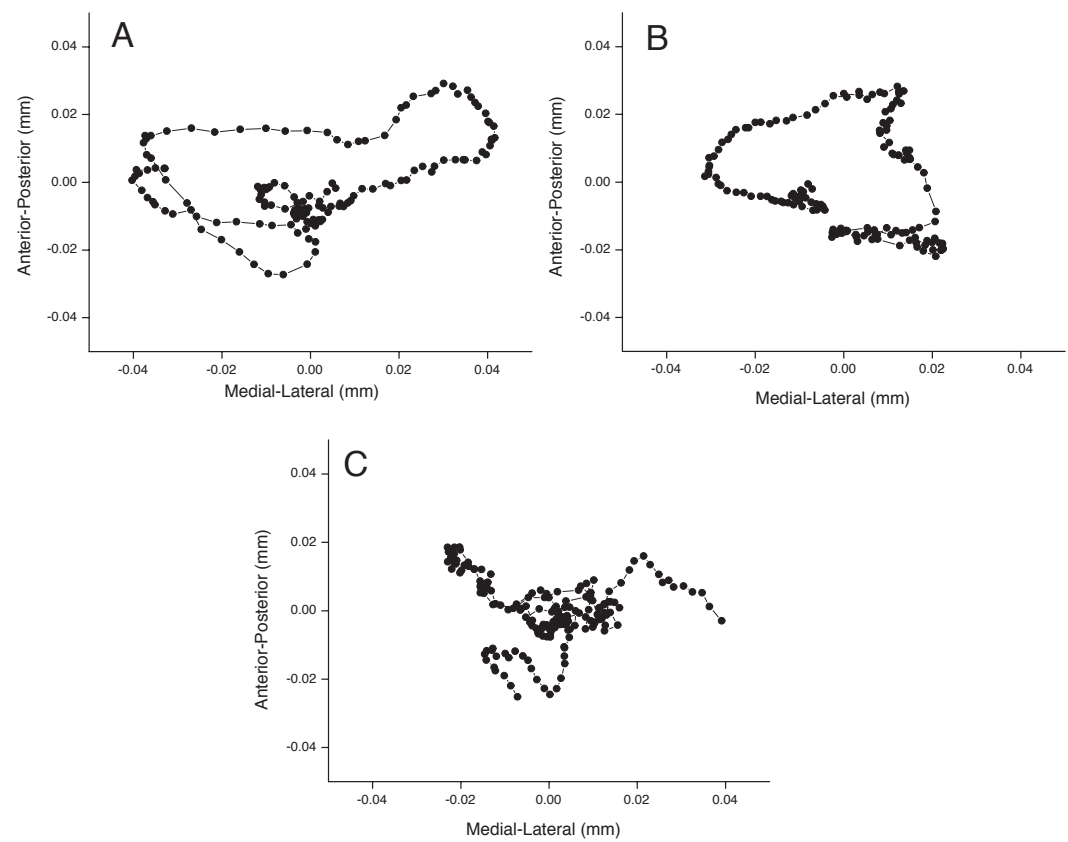

Figure 3: Direction of centre of pressure during a single trial of squatting during each condition; flat shoes $(A)$, bare feet $(B)$, and weightlifting shoes $(C)$. 
Condition

Novice

$\begin{array}{cccccccc}\text { Bare Feet } & 34.75 & 48.25 & 34.25 & 18.13 & 4.25 & 0.75 & 140.38 \\ \quad \text { Flat Shoes } & 42.25 & 64.50 & 31.63 & 10.13 & 3.50 & 2.38 & 154.38 \\ \quad \begin{array}{c}\text { Weightlifting Shoes } \\ \text { Advanced }\end{array} & 27.63 & 72.75 & 35.13 & 14.88 & 6.50 & 6.25 & 163.13 \\ \quad \text { Bare Feet } & 41.38 & 52.00 & 30.50 & 16.38 & 2.88 & 0.38 & 135.50 \\ \quad \text { Flat Shoes } & 49.00 & 62.88 & 22.50 & 6.13 & 2.50 & 2.50 & 143.50 \\ \text { Weightlifting Shoes } & 27.63 & 72.75 & 35.125 & 14.88 & 6.50 & 6.25 & 145.50 \\ \text { Total } & 221.25 & 361.88 & 188.63 & 76.38 & 21.50 & 12.75 & 882.38\end{array}$

Observed Values
Radius (mm)

10

20

30

40

50

60

Total

Table 1: Chi-square Observed Values (degrees of freedom $=25)$ for Novice and Advanced Participants

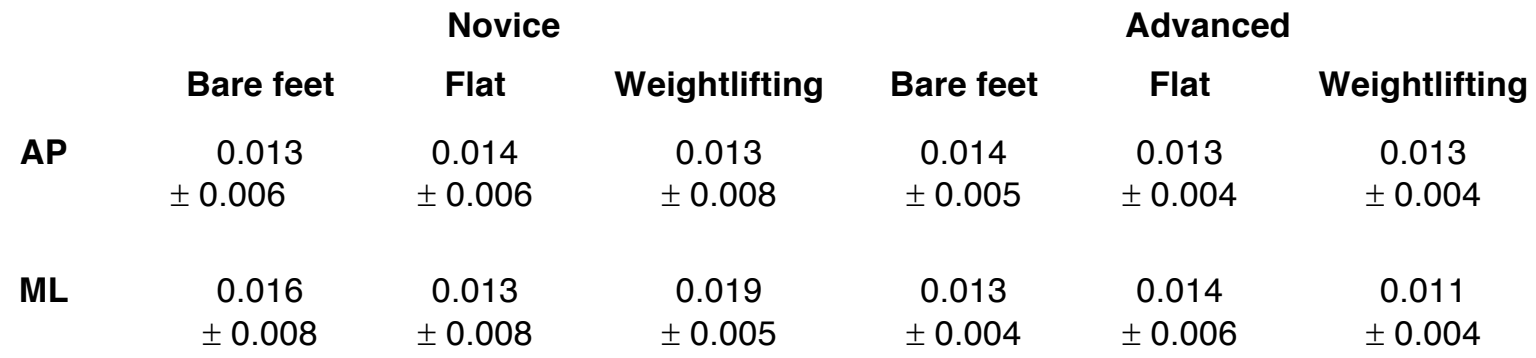

Table 2: RMS Mean and Standard Deviations in AP and ML Directions for Novice and Advanced Participants. $\mathrm{AP}$ - Anterior-Posterior; ML - Medial-Lateral.

\section{Discussion}

The purpose of the study was to determine the differences in balance regulation between BF, FS, and WLS conditions, in novice and advanced participants. It was hypothesized that WLS would regulate balance more effectively than the other footwear conditions. The WLS condition elicited improved balance performance compared to BF and FS. CoPs was centralized toward the middle their BoS (Figure 3). It was also hypothesized that advanced participants would demonstrate increased balance performance in the $\mathrm{AP}$ and $\mathrm{ML}$ directions compared to novice participants. This was not supported, as there was no statistically significant difference between novice and advanced participants in balance regulation.

The 2-way ANOVA found no significant differences between the comparison groups and the specific footwear condition (Table 2); experience level had no effect on balance performance when wearing the same footwear. Balance is regulated at the highest order of cognitive function in healthy individuals (16). It takes priority over noxious stimuli, cognitive function, and movement speed $(16,17,18)$. Dual tasks, with balancing and cognitive components, show diminishing results in the cognitive tasks in order to maintain balance (17). Furthermore, movement speed demonstrates a similar effect; speed decreases in order to regulate balance (18). Squatting experience levels appear to make no difference in regulating balance during a squat movement; novice squatters may have decreased cognitive function or movement speed; however, their balance will remain regulated. Regardless of familiarity with the squat movement, because it is a simple task, individuals will maintain balance. Since the sample population was comprised of able-bodied individuals, this is likely the reason for the lack of difference in balance regulation.

As mentioned earlier, balance is regulated by the CNS and postural changes, indicating an array of explanations for the differences found between the footwear conditions. Previous research has 
established that bare feet and barefoot-inspired shoes have similar characteristics in regulating balance (19). Similar to the present findings, there were no significant differences between $\mathrm{BF}$ and $\mathrm{FS}$ conditions. These conditions require similar kinematic adjustments as the foot positioning is the same on a non-compressible surface. Presumably, this allows for similar muscular recruitment and activation throughout the squat movement. These similarities are noted by the lack of differences between the percentage of time spent in each radius within the BoS. The main difference between the 2 conditions is that FS is thought to provide less afferent cutaneous information from the plantar aspect of the foot. As previously mentioned, balance is regulated by three afferent systems. As vestibular and visual information is consistent between the conditions, the comparison illustrates the effectiveness of the cutaneous system. The insignificant differences between FS and BF is indicative of cutaneous information not being heavily relied upon in balance regulation during a squat movement. As the cutaneous system is impaired, the vestibular and visual systems accommodate the deficiency, and balance is maintained (9). To maintain safety, many gyms require patrons to wear indoor footwear. This protects patrons from injury, and prevents the spread of bacteria. As participants during the BF and FS conditions regulate balance similarly, patrons can utilize FS while adhering to gym rules and maintaining safety.

While there were no statistically significant differences between participants in the BF and FS condition, it was found that WLS are superior in balance regulation. CoP in WLS had a larger percentage of time spent in a $10 \mathrm{~mm}$ and $20 \mathrm{~mm}$ radii within the BoS. The main difference between WLS and the other conditions is the addition of a 1.5" heel lift. Since increasing the plantar flexion angle increases plantar flexor activation, adding a 1.5" heel lift will have the same effect (13). As the AP balance is regulated by the plantar flexors, it was postulated that increasing plantar flexor activation will increase the effectiveness of the ankle strategy. Furthermore, kinematic changes at the ankles will influence adjustments at the hip. Previous studies suggest that WLS reduce the amount of trunk lean, caused by greater hip extensor moments (12). This results in a safer squat technique as less shear forces are applied to the lower back.

The present findings, specifically the superiority of WLS, can help individuals build lower limb strength, while mitigating the risk of falling and injury. Specific populations, such as stroke patients, are at a higher risk of falling after damaging their balance regulation pathways (19). With the aid of WLS, these patients can utilize the strengthening benefits of the squat, while minimizing the risk of falling.

\section{Limitations and Future Directions}

The population of the study was solely male. Fundamentally, there are anatomical differences between females and males. Females tend to have wider pelves and greater muscle flexibility, contributing to differences in squat mechanics (20, 21). In addition, females are more likely to wear highheeled shoes, potentially playing a role in acclimatization to the WLS heel lift. Future studies should include participants with a broader range of ages and includes both sexes to determine if results differ across populations.

The classification of advanced vs novice squatters does not necessarily reflect their skill sets. As the classification of each condition was based upon the frequency of squatting rather than technique and total time squatting. In future studies, squatting technique should be analyzed and used to demarcate groups accordingly.

The amount of experience participants' had in each footwear condition was different. Participants could be comfortable squatting with specific footwear, possibly leading to better balance performance in their preferred footwear condition. To achieve more homogeneous results in future studies, there should be congruency within the groups based on the level of comfort with each footwear conditions.

The protocol required participants to squat with an unweighted barbell. Kinematic and kinetic changes in squat occur as weight is increased (14). Adding weight causes changes in the musculature recruitment, and thus potential changes in balance regulation. In future studies, the weight of the barbell used should be adjusted accordingly for the advanced and the novice groups.

Joint angle and muscle activation patterns were not measured. Future studies should consider spatial-temporal parameters, through motion capture, and electromyography to quantify changes in musculature recruitment.

\section{Conclusion}

The results of this study demonstrate that participants during the WLS condition regulated balance better than during BF and FS conditions. 
The initial plantar flexion caused by the WLS heel lift activated plantar flexors for the ankle strategy, resulting in greater balance performance. Furthermore, it was determined that the cutaneous system had minimal effects on balance during a squat, and thus balance performance in the BF and FS conditions were similar.

Additionally, it was found that advanced participants did not have increased balance performance compared to novice participants. As balance takes the highest priority in cognitive function, individuals have similar balance performances regardless of experience level.

The findings of the present study are applicable for resistance training and rehabilitation. By using WLS, an individual has increased balance performance, thereby mitigating the risk of falling and injury during squat movements.

\section{References}

1. Ada L, Dorsch S, Canning CG. Strengthening interventions increase strength and improve activity after stroke: a systematic review. Aust J Physiother. 2006;52(4):241-248.

2. Walsh JC, Quinlan JF, Stapleton R, FitzPatrick D P, McCormack D. Three-dimensional motion analysis of the lumbar spine during "Free squat" weight lift training. Am $J$ Sports Med. 2007 Jun;35(6):927-932.

3. Lee TS, Song MY, Kwon YJ. Activation of back and lower limb muscles during squat exercises with different trunk flexion. $J$ Phys Ther Sci. 2016 Dec;28(12):3407-3410.

4. Frost LR, Brown SH. Muscle activation timing and balance response in chronic lower back pain patients with associated radiculopathy. Clin Biomech. 2016 Feb;32:124-30.

5 . Winter DA. Human balance and posture control during standing and walking. Gait Posture. 1995 Dec;3(4):193-214.

6. Woollacott MH, Pei-Fang T. Balance control during walking in the older adult: research and its implications. Phys ther. 1997 Jun;77(6):646.

7. Horak, F. B. Postural orientation and equilibrium: what do we need to know about neural control of balance to prevent falls? Age and ageing. 2016 Jun; 3

8. Nashner LM, McCollum G. The organization of human postural movements: a formal basis and experimental synthesis. $J$ Behav Brain Sci. 1985 Mar;8(01):135-150.

9. Fitzpatrick R, McCloskey DI. Proprioceptive, visual and vestibular thresholds for the perception of sway during standing in humans. Journal Physiol. 1995 Jul;478(Pt 1):173.

10. Magnusson $M$, Enbom $H$, Johansson $R$, Wiklund $J$. Significance of pressor input from the human feet in lateral postural control: The effect of hypothermia on galvanically induced body-sway. Acta Otolaryngol. 1990 Nov;110(56):321-327.

11. Lynn S, Noffal GJ, Pittman MB. Hip and Knee Moments During Regular and Counter-Balance Squats. J Strength Cond Res. 2011 Mar;25:S21-S22.

12. Sato K, Fortenbaugh D, Hydock DS. Kinematic changes using weightlifting shoes on barbell back squat. J Strength Cond Res. 2012 Jan;26(1):28-33.

13. Ki KI, Choi JD, Cho HS. The effect of ground tilt on the lower extremity muscle activity of stroke patients performing squat exercises. J Phys Ther Sci. 2014 Jul;26(7):965-968
14. Sinclair J, McCarthy D, Bentley I, Hurst HT, Atkins S. The influence of different footwear on 3-D kinematics and muscle activation during the barbell back squat in males. Eur J Sport Sci. 2015;15(7):583-590.

15. Whitting JW, Meir RA, Crowley-McHattan ZJ, Holding RC. Influence of Footwear Type on Barbell Back Squat Using 50, 70 , and $90 \%$ of One Repetition Maximum: A Biomechanical Analysis. J Strength Cond Res. 2016 Apr;30(4):1085-1092.

16. Lee RG, Tonolli I, Viallet F, Aurenty R, Massion J. Preparatory postural adjustments in parkinsonian patients with postural instability. Can J Neurol Sci. 1995 May;22(02):126-135.

17. Muhaidat J, Kerr A, Evans JJ, Pilling M, Skelton DA. Validity of simple gait-related dual-task tests in predicting falls in community-dwelling older adults. Arch Phys Med Rehabil. 2014 Jan;95(1):58-64.

18. Wall JC, Bell C, Campbell S, Davis J. The Timed Get-up-andGo test revisited: measurement of the component tasks. J Rehabil Res Dev. 2007 Jan;37(1):109.

19. Peters S, Handy TC, Lakhani B, Boyd LA, Garland SJ. Motor and Visuospatial Attention and Motor Planning After Stroke: Considerations for the Rehabilitation of Standing Balance and Gait. Phys Ther. 2015 Apr;95(10):1423.

20. Horan SA, Evans K, Morris NR, Kavanagh JJ. Thorax and pelvis kinematics during the downswing of male and female skilled golfers. J Biomech. 2010 May;43(8):1456-1462.

21. Hale R, Hausselle JG, Gonzalez RV. A preliminary study on the differences in male and female muscle force distribution patterns during squatting and lunging maneuvers. Comput Biol Med. 2014 Sep;52:57-65. 